\title{
STANLEY CONJECTURE ON MONOMIAL IDEALS OF MIXED PRODUCTS
}

\author{
GAETANA RESTUCCIA, ZHONGMING TANG AND ROSANNA UTANO
}

\begin{abstract}
It is proved that the Stanley conjecture holds for monomial ideals of mixed products, i.e., if $I$ is an ideal of mixed products in a polynomial ring $S$ over a field, then $\operatorname{sdepth}_{S}(I) \geq \operatorname{depth}_{S}(I)$ and $\operatorname{sdepth}_{S}(S / I) \geq$ $\operatorname{depth}_{S}(S / I)$.
\end{abstract}

1. Introduction. Let $S=K\left[x_{1}, \ldots, x_{n}\right]$ be the polynomial ring in $n$ variables over a field $K$ and $M$ a finitely generated $\mathbb{Z}^{n}$-graded $S$ module. A Stanley decomposition $\mathcal{D}$ of $M$ is a finite direct sum of $K$-spaces:

$$
\mathcal{D}: M=\bigoplus_{i=1}^{r} m_{i} K\left[Z_{i}\right] \text {, }
$$

where $m_{i} \in M$ is homogeneous and $Z_{i} \subseteq\left\{x_{1}, \ldots, x_{n}\right\}, i=1, \ldots, r$, and its Stanley depth, $\operatorname{sdepth}_{S}(\mathcal{D})$, is defined as $\min \left\{\left|Z_{i}\right| \mid i=1, \ldots, r\right\}$. By definition, the Stanley depth of $M$ is the following

$\operatorname{sdepth}_{S}(M)=\max \left\{\operatorname{sdepth}_{S}(\mathcal{D}) \mid \mathcal{D}\right.$ is a Stanley decomposition of $\left.M\right\}$.

Stanley [13] conjectured that $\operatorname{sdepth}_{S}(M) \geq \operatorname{depth}_{S}(M)$. There has been much research on this conjecture, especially when $M$ has the form $S / I$ or $I$ with $I$ a square-free monomial ideal of $S$, cf., $[\mathbf{8 , 9}, \mathbf{1 0}, \mathbf{1 4}]$. In this paper, we consider the case where $I$ is an ideal of mixed products.

Let $S=K\left[x_{1}, \ldots, x_{n}, y_{1}, \ldots, y_{m}\right], S_{1}=K\left[x_{1}, \ldots, x_{n}\right]$ and $S_{2}=$ $K\left[y_{1}, \ldots, y_{m}\right]$ be polynomial rings over $K$. Let $I \subset I^{\prime} \subset S_{1}$ and

2010 AMS Mathematics subject classification. Primary 13C15, 13F20.

The authors would like to thank INDAM (Istituto Nazionale di Alta Matematica "F. Severi," Roma, Italy) for financial support. The second author is grateful to the Natural Science Foundation of Jiangsu Province and the National Natural Science Foundation of China for partial support. The second author is the corresponding author.

Received by the editors on August 8, 2013, and in revised form on October 30, 2013. 
$J \subset J^{\prime} \subset S_{2}$ be non zero monomial ideals. We call a generalized mixed product of ideals the ideal defined as

$$
L=\left(I^{\prime} J+I J^{\prime}\right) S .
$$

If $I=I_{q}$ and $I^{\prime}=I_{r}$ are square-free Veronese monomial ideals in $S_{1}$, and $J=J_{s}$ and $J^{\prime}=J_{t}$ are square-free Veronese monomial ideals in $S_{2}$, i.e., they are generated by all the square-free monomials of degree $q, r$, $s$ and $t$, respectively, we recover the class of ideals of mixed products, as defined in [12]. In particular, if the ideal $L$ has the form $I_{r} J_{r}$ or $I_{r} J_{r-1}+I_{r-1} J_{r}, L$ is the generalized graph ideal of a complete bipartite graph whose vertices are $x_{1}, \ldots, x_{n}, y_{1}, \ldots, y_{n}([\mathbf{1 2}])$. From the geometric point of view we can look at $L$ as the ideal $I_{\Delta}$ where $\Delta$ is a simplicial complex and $I_{\Delta}$ is its Stanley-Reisner ideal. In particular, for $L=I_{r}+J_{r}$, as before, the structure of the simplicial complex $\Delta$ is not difficult to understand, being the join $\Delta_{1} * \Delta_{2}$ of two disjoint simplicial complexes consisting of the $(r-1)$-skeletons of two $(r-1)$-simplices on the sets of vertices $\left\{x_{1}, \ldots, x_{n}\right\}$ and $\left\{y_{1}, \ldots, y_{n}\right\}$, respectively, with $I_{r}=I_{\Delta_{1}}$ and $J_{r}=I_{\Delta_{2}}$. We refer to $[\mathbf{1}, \mathbf{1 5}]$ for basic properties of Stanley-Reisner ideals.

Section 2 consists of background material on depth and Stanley depth of ideals $I S+J S, I S \cap J S, S /(I S+J S)$ and $S /(I S \cap J S)$. In Section 3, we give lower bounds for the Stanley depth of ideals of generalized mixed products $L=\left(I^{\prime} J+I J^{\prime}\right) S$ and of $S / L$, under some hypotheses. These assumptions are not strong, since they are verified for large classes of ideals (for example, in the Veronese square-free case). In Section 4, the Stanley depth conjecture is verified for the class of ideals of mixed products as defined in $[\mathbf{5}, \mathbf{1 2}]$.

2. Preliminaries. Let $S=K\left[x_{1}, \ldots, x_{n}, y_{1}, \ldots, y_{m}\right], S_{1}=K\left[x_{1}\right.$, $\left.\ldots, x_{n}\right]$ and $S_{2}=K\left[y_{1}, \ldots, y_{m}\right]$. For $1 \leq q \leq n$ (respectively, $1 \leq p \leq m$ ), denote the ideal of $S_{1}$ (respectively, $S_{2}$ ) generated by all the square-free monomials of degree $q$ (respectively, $p$ ) by $I_{q}$ (respectively, $J_{p}$ ).

Lemma 2.1. ([5, Theorems 3.4 and 3.7]). Let $1 \leq q<s, 1 \leq t<r$. Then

(1) $\operatorname{depth}_{S}\left(S / I_{q} S\right)=m+q-1, \operatorname{depth}\left(S / J_{t} S\right)=n+t-1$;

(2) $\operatorname{depth}_{S}\left(S /\left(I_{q} J_{t}\right) S\right)=q+t-1$; 
(3) $\operatorname{depth}_{S}\left(S /\left(I_{q}+J_{t}\right) S\right)=q+t-2$;

(4) $\operatorname{depth}_{S}\left(S /\left(I_{q} J_{r}+I_{s}\right) S\right)=q+r-1$;

(5) $\operatorname{depth}_{S}\left(S /\left(I_{q} J_{r}+I_{s} J_{t}\right) S\right)=\min \{q+r, s+t\}-1$.

The following lemma is a result observed in the proof of [3, Theorem 3.1].

Lemma 2.2. Let $H$ be a multigraded $S_{1}$-module and $L$ a multigraded $S_{2}$-module. Then, as multigraded $S$-modules,

$$
\operatorname{sdepth}_{S}\left(H \otimes_{K} L\right) \geq \operatorname{sdepth}_{S_{1}}(H)+\operatorname{sdepth}_{S_{2}}(L) .
$$

For the Stanley depth, we have the following

Lemma 2.3. Let $I \subset S_{1}$ and $J \subset S_{2}$ be monomial ideals. Then

(1) $\left(\left[\mathbf{2}\right.\right.$, Theorem 1.3]). $\operatorname{sdepth}_{S}(I S+J S) \geq \min \left\{\operatorname{sdepth}_{S}(I S)\right.$, $\left.\operatorname{sdepth}_{S_{2}}(J)+\operatorname{sdepth}_{S_{1}}\left(S_{1} / I\right)\right\}$

(2) $\left(\left[\mathbf{7}, \operatorname{Lemma}_{1.2])} . \operatorname{sdepth}_{S}(I S \cap J S) \geq \operatorname{sdepth}_{S_{1}}(I)+\operatorname{sdepth}_{S_{2}}(J)\right.\right.$;

(3) $\left(\left[\mathbf{1 1}\right.\right.$, Theorem 3.1]). $\operatorname{sdepth}_{S}(S /(I S+J S)) \geq \operatorname{sdepth}_{S_{1}}\left(S_{1} / I\right)+$ $\operatorname{sdepth}_{S_{2}}\left(S_{2} / J\right)$

(4) ([2, Theorem 1.3]). $\operatorname{sdepth}_{S}(S /(I S \cap J S)) \geq \min \left\{\operatorname{sdepth}_{S}(S / I S)\right.$, $\left.\operatorname{sdepth}_{S_{1}}(I)+\operatorname{sdepth}_{S_{2}}\left(S_{2} / J\right)\right\}$

(5) $\operatorname{sdepth}_{S}(I S /(I S \cap J S)) \geq \operatorname{sdepth}_{S_{1}}(I)+\operatorname{sdepth}_{S_{2}}\left(S_{2} / J\right)$.

Proof. For item (5), note that $I S \cap J S=(I J) S$ and $I S /(I S \cap J S) \cong$ $I \otimes_{K} S_{2} / J$ as $K$-spaces. Then the result follows from Lemma 2.2.

From [4, Proposition 3.1], we have

\section{Lemma 2.4.}

(1) $\operatorname{sdepth}_{S_{1}}\left(S_{1} / I_{q}\right)=q-1$;

(2) Let $I=\left(u_{1}, \ldots, u_{r}\right)$ be a square-free monomial ideal of $S_{1}$. Then

$$
\operatorname{sdepth}_{S_{1}}(I) \geq \min \left\{\operatorname{deg}\left(u_{i}\right) \mid i=1, \ldots, r\right\} .
$$

Then, by [4, Lemma 3.6], $\operatorname{sdepth}_{S}\left(S / I_{q} S\right)=m+q-1$ and $\operatorname{sdepth}_{S}(I S)=m+\operatorname{sdepth}_{S_{1}}(I), \operatorname{especially}_{\operatorname{sdepth}_{S}}\left(I_{q} S\right) \geq m+q$. 
3. Stanley depths of generalized mixed products of ideals. Let $I \subset I^{\prime} \subset S_{1}$ and $J \subset J^{\prime} \subset S_{2}$ be nonzero monomial ideals. We call the following ideal of $S$ as a generalized mixed product of ideals:

$$
\left(I^{\prime} J+I J^{\prime}\right) S \text {. }
$$

For the generalized mixed products of ideals, the following four propositions estimate their Stanley depths.

\section{Proposition 3.1.}

(1) $\operatorname{sdepth}_{S}((I J) S) \geq \operatorname{sdepth}_{S_{1}}(I)+\operatorname{sdepth}_{S_{2}}(J)$;

$(2) \operatorname{sdepth}_{S}(S /(I J) S) \geq \operatorname{sdepth}_{S_{2}}\left(S_{2} / J\right)+\min \left\{\operatorname{sdepth}_{S_{1}}(I)\right.$, $\left.\operatorname{sdepth}_{S_{1}}\left(S_{1} / I\right)+1\right\}$.

Proof.

(1) Since $(I J) S=I S \cap J S$, it follows from Lemma 2.3 (2).

(2) Firstly, note that $\operatorname{sdepth}_{S_{2}}\left(S_{2} / J\right) \neq m$; otherwise, $S_{2} / J=u S_{2}$ for some monomial $u$. If $u=1$, then $J=0$, and if $u \neq 1$, then $1 \in J$, a contradiction. Then, by Lemma 2.3 (4), we have

$$
\begin{gathered}
\operatorname{sdepth}_{S}(S /(I J) S)=\operatorname{sdepth}_{S}(S /(I S \cap J S)) \\
\geq \min \left\{\operatorname{sdepth}_{S_{1}}\left(S_{1} / I\right)+m,\right. \\
\left.\operatorname{sdepth}_{S_{2}}\left(S_{2} / J\right)+\operatorname{sdepth}_{S_{1}}(I)\right\} \\
\geq \min \left\{\operatorname{sdepth}_{S_{1}}\left(S_{1} / I\right)+\operatorname{sdepth}_{S_{2}}\left(S_{2} / J\right)+1,\right. \\
\left.\operatorname{sdepth}_{S_{2}}\left(S_{2} / J\right)+\operatorname{sdepth}_{S_{1}}(I)\right\} \\
=\operatorname{sdepth}_{S_{2}}\left(S_{2} / J\right)+\min \left\{\operatorname{sdepth}_{S_{1}}(I),\right. \\
\left.\operatorname{sdepth}_{S_{1}}\left(S_{1} / I\right)+1\right\} .
\end{gathered}
$$

Proposition 3.2. $\operatorname{sdepth}_{S}((I+J) S) \geq \operatorname{sdepth}_{S_{2}}(J)+\min \left\{\operatorname{sdepth}_{S_{1}}(I)\right.$, $\left.\operatorname{sdepth}_{S_{1}}\left(S_{1} / I\right)\right\}$.

Proof. By virtue of Lemma 2.3 (1), one gets

$$
\begin{aligned}
& \operatorname{sdepth}_{S}((I+J) S) \geq \min \left\{\operatorname{sdepth}_{S_{1}}(I)+m, \operatorname{sdepth}_{S_{2}}(J)\right. \\
& \left.\quad+\operatorname{sdepth}_{S_{1}}\left(S_{1} / I\right)\right\} \\
& \geq \min \left\{\operatorname{sdepth}_{S_{1}}(I)+\operatorname{sdepth}_{S_{2}}(J), \operatorname{sdepth}_{S_{2}}(J)\right. \\
& \left.\quad+\operatorname{sdepth}_{S_{1}}\left(S_{1} / I\right)\right\}
\end{aligned}
$$




$$
=\operatorname{sdepth}_{S_{2}}(J)+\min \left\{\operatorname{sdepth}_{S_{1}}(I), \operatorname{sdepth}_{S_{1}}\left(S_{1} / I\right)\right\} .
$$

\section{Proposition 3.3.}

(1) Suppose that $\operatorname{sdepth}_{S_{2}}(J)>\operatorname{sdepth}_{S_{2}}\left(S_{2} / J\right)$. Then

$$
\begin{aligned}
& \operatorname{sdepth}_{S}\left(\left(I^{\prime} J+I\right) S\right) \geq \operatorname{sdepth}_{S_{2}}\left(S_{2} / J\right) \\
& \quad+\min \left\{\operatorname{sdepth}_{S_{1}}\left(I^{\prime}\right)+1, \operatorname{sdepth}_{S_{1}}(I)\right\} .
\end{aligned}
$$

(2) Suppose that $\operatorname{sdepth}_{S_{1}}\left(S_{1} / I\right)>\operatorname{sdepth}_{S_{1}}\left(S_{1} / I^{\prime}\right)$. Then

$$
\begin{aligned}
& \operatorname{sdepth}_{S}\left(S /\left(I^{\prime} J+I\right) S\right) \geq \operatorname{sdepth}_{S_{1}}\left(S_{1} / I^{\prime}\right) \\
& \quad+\min \left\{\operatorname{sdepth}_{S_{2}}(J), \operatorname{sdepth}_{S_{2}}\left(S_{2} / J\right)+1\right\} .
\end{aligned}
$$

Proof.

(1) As $K$-spaces, we have the following decompositions

$$
\begin{aligned}
\left(I^{\prime} J+I\right) S & \cong\left(I^{\prime} J\right) S \oplus \frac{\left(I^{\prime} J+I\right) S}{\left(I^{\prime} J\right) S} \\
& \cong\left(I^{\prime} J\right) S \oplus \frac{I S}{\left(I^{\prime} J\right) S \cap I S} \\
& =\left(I^{\prime} J\right) S \oplus \frac{I S}{I S \cap J S \cap I^{\prime} S} \\
& =\left(I^{\prime} J\right) S \oplus \frac{I S}{I S \cap J S} .
\end{aligned}
$$

It follows from Lemma $2.3(5)$ that

$$
\begin{aligned}
& \operatorname{sdepth}_{S}\left(\left(I^{\prime} J+I\right) S\right) \\
& \geq \min \left\{\operatorname{sdepth}_{S}\left(\left(I^{\prime} J\right) S\right), \operatorname{sdepth}_{S}(I S /(I S \cap J S))\right\} \\
& \geq \min \left\{\operatorname{sdepth}_{S_{1}}\left(I^{\prime}\right)+\operatorname{sdepth}_{S_{2}}(J), \operatorname{sdepth}_{S_{1}}(I)\right. \\
& \left.\quad+\operatorname{sdepth}_{S_{2}}\left(S_{2} / J\right)\right\} .
\end{aligned}
$$

Under the assumption that $\operatorname{sdepth}_{S_{2}}(J)>\operatorname{sdepth}_{S_{2}}\left(S_{2} / J\right)$, it turns out that

$$
\begin{aligned}
& \operatorname{sdepth}_{S}\left(\left(I^{\prime} J+I\right) S\right) \geq \operatorname{sdepth}_{S_{2}}\left(S_{2} / J\right) \\
& \quad+\min \left\{\operatorname{sdepth}_{S_{1}}\left(I^{\prime}\right)+1, \operatorname{sdepth}_{S_{1}}(I)\right\} .
\end{aligned}
$$


(2) As $K$-spaces, we have the following decompositions

$$
\begin{aligned}
S /\left(I^{\prime} J+I\right) S & \cong \frac{S}{(J+I) S} \oplus \frac{(J+I) S}{\left(I^{\prime} J+I\right) S} \\
& =\frac{S}{(J+I) S} \oplus \frac{(J+I) S}{I^{\prime} S \cap(J+I) S} \\
& \cong \frac{S}{(J+I) S} \oplus \frac{\left(I^{\prime}+J+I\right) S}{I^{\prime} S} \\
& =\frac{S}{(J+I) S} \oplus \frac{\left(I^{\prime}+J\right) S}{I^{\prime} S} \\
& \cong \frac{S}{(J+I) S} \oplus \frac{J S}{I^{\prime} S \cap J S} .
\end{aligned}
$$

Then, it follows from Lemma 2.3 (3) and (5) that

$$
\begin{aligned}
& \operatorname{sdepth}_{S}\left(S /\left(I^{\prime} J+I\right) S\right) \\
& \geq \min \left\{\operatorname{sdepth}_{S}(S /(J+I) S),\right. \\
& \left.\operatorname{sdepth}_{S}\left(J S /\left(I^{\prime} S \cap J S\right)\right)\right\} \\
& \geq \min \left\{\operatorname{sdepth}_{S_{1}}\left(S_{1} / I\right)+\operatorname{sdepth}_{S_{2}}\left(S_{2} / J\right),\right. \\
& \left.\operatorname{sdepth}_{S_{2}}(J)+\operatorname{sdepth}_{S_{1}}\left(S_{1} / I^{\prime}\right)\right\} .
\end{aligned}
$$

If $\operatorname{sdepth}_{S_{1}}\left(S_{1} / I\right)>\operatorname{sdepth}_{S_{1}}\left(S_{1} / I^{\prime}\right)$, then

$$
\begin{aligned}
& \operatorname{sdepth}_{S}\left(S /\left(I^{\prime} J+I\right) S\right) \geq \operatorname{sdepth}_{S_{1}}\left(S_{1} / I^{\prime}\right) \\
& \quad+\min \left\{\operatorname{sdepth}_{S_{2}}(J), \operatorname{sdepth}_{S_{2}}\left(S_{2} / J\right)+1\right\} .
\end{aligned}
$$

\section{Proposition 3.4.}

(1) $\operatorname{sdepth}_{S}\left(\left(I^{\prime} J+I J^{\prime}\right) S\right) \geq \min \left\{\operatorname{sdepth}_{S_{1}}(I)+\operatorname{sdepth}_{S_{2}}\left(J^{\prime}\right)\right.$, $\left.\operatorname{sdepth}_{S_{1}}\left(I^{\prime} / I\right)+\operatorname{sdepth}_{S_{2}}(J)\right\}$

(2) Suppose that $\operatorname{sdepth}_{S_{1}}\left(S_{1} / I\right)>\operatorname{sdepth}_{S_{1}}\left(S_{1} / I^{\prime}\right)$. Then

$$
\begin{aligned}
& \operatorname{sdepth}_{S}\left(S /\left(I^{\prime} J+I J^{\prime}\right) S\right) \\
& \geq \min \left\{\operatorname{sdepth}_{S_{1}}\left(S_{1} / I^{\prime}\right)+\min \left\{\operatorname{sdepth}_{S_{2}}(J),\right.\right. \\
& \left.\operatorname{sdepth}_{S_{2}}\left(S_{2} / J\right)+1\right\}, \operatorname{sdepth}_{S_{1}}(I) \\
& \left.+\operatorname{sdepth}_{S_{2}}\left(S_{2} / J^{\prime}\right)\right\} .
\end{aligned}
$$


Proof.

(1) As $K$-spaces, we have the following decompositions

$$
\begin{aligned}
\left(I^{\prime} J+I J^{\prime}\right) S & \cong\left(I J^{\prime}\right) S \oplus \frac{\left(I^{\prime} J+I J^{\prime}\right) S}{\left(I J^{\prime}\right) S} \\
& \cong\left(I J^{\prime}\right) S \oplus \frac{\left(I^{\prime} J\right) S}{I S \cap J^{\prime} S \cap I^{\prime} S \cap J S} \\
& =\left(I J^{\prime}\right) S \oplus \frac{\left(J I^{\prime}\right) S}{(J I) S} \\
& \cong\left(I J^{\prime}\right) S \oplus\left(J \otimes_{K} \frac{I^{\prime}}{I}\right) S .
\end{aligned}
$$

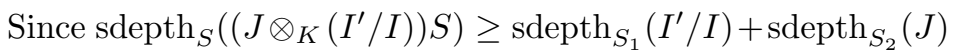
by Lemma 2.2 , it follows that

$$
\begin{aligned}
& \operatorname{sdepth}_{S}\left(\left(I^{\prime} J+I J^{\prime}\right) S\right) \\
& \geq \min \left\{\operatorname{sdepth}_{S}\left(\left(I J^{\prime}\right) S\right), \operatorname{sdepth}_{S}\left(\left(J \otimes_{K} \frac{I^{\prime}}{I}\right) S\right)\right\} \\
& \geq \min \left\{\operatorname{sdepth}_{S_{1}}(I)+\operatorname{sdepth}_{S_{2}}\left(J^{\prime}\right),\right. \\
& \left.\operatorname{sdepth}_{S_{1}}\left(I^{\prime} / I\right)+\operatorname{sdepth}_{S_{2}}(J)\right\} .
\end{aligned}
$$

(2) As $K$-spaces, the following decompositions hold

$$
\begin{aligned}
S /\left(I^{\prime} J+I J^{\prime}\right) S & \cong \frac{S}{\left(I^{\prime} J+I\right) S} \oplus \frac{\left(I^{\prime} J+I\right) S}{\left(I^{\prime} J+I J^{\prime}\right) S} \\
& =\frac{S}{\left(I^{\prime} J+I\right) S} \oplus \frac{\left(I^{\prime} J+I\right) S}{\left(I^{\prime} J+I\right) S \cap J^{\prime} S} \\
& \cong \frac{S}{\left(I^{\prime} J+I\right) S} \oplus \frac{\left(I^{\prime} J+I\right) S+J^{\prime} S}{J^{\prime} S} \\
& =\frac{S}{\left(I^{\prime} J+I\right) S} \oplus \frac{\left(I+J^{\prime}\right) S}{J^{\prime} S} \\
& \cong \frac{S}{\left(I^{\prime} J+I\right) S} \oplus \frac{I S}{I S \cap J^{\prime} S} .
\end{aligned}
$$

By the assumption that $\operatorname{sdepth}_{S_{1}}\left(S_{1} / I\right)>\operatorname{sdepth}_{S_{1}}\left(S_{1} / I^{\prime}\right)$, from Proposition 3.3 (2), we have

$$
\begin{aligned}
& \operatorname{sdepth}_{S}\left(S /\left(I^{\prime} J+I J^{\prime}\right) S\right) \\
& \quad \geq \min \left\{\operatorname{sdepth}_{S}\left(S /\left(I^{\prime} J+I\right) S\right),\right.
\end{aligned}
$$




$$
\begin{gathered}
\left.\operatorname{sdepth}_{S}\left(I S /\left(I S \cap J^{\prime} S\right)\right)\right\} \\
\geq \min \left\{\operatorname{sdepth}_{S_{1}}\left(S_{1} / I^{\prime}\right)\right. \\
+\min \left\{\operatorname{sdepth}_{S_{2}}(J), \operatorname{sdepth}_{S_{2}}\left(S_{2} / J\right)+1\right\} \\
\left.\operatorname{sdepth}_{S_{1}}(I)+\operatorname{sdepth}_{S_{2}}\left(S_{2} / J^{\prime}\right)\right\}
\end{gathered}
$$

4. Stanley conjecture on ideals of mixed products. Let $S=$ $K\left[x_{1}, \ldots, x_{N}\right]$ be a polynomial ring over a field $K$ and $L$ a square-free monomial ideal of $S$. We say that $L$ is an ideal of mixed products if there exists an integer $n$ such that $1 \leq n<N$ and

$$
L=\left(I_{q} J_{r}+I_{s} J_{t}\right) S, \quad 0 \leq q<s \leq n, 0 \leq t<r \leq N-n,
$$

where $I_{q}$ (respectively, $I_{s}$ ) is the square-free monomial ideal of $K\left[x_{1}\right.$, $\ldots, x_{n}$ ] generated by all the square-free monomials of degree $q$ (respectively, $s$ ), and $J_{r}$ (respectively, $J_{t}$ ) is the square-free monomial ideal of $K\left[x_{n+1}, \ldots, x_{N}\right]$ generated by all the square-free monomials of degree $r$ (respectively, $t$ ). We use the convention that $I_{0}=K\left[x_{1}, \ldots, x_{n}\right]$ and $J_{0}=K\left[x_{n+1}, \ldots, x_{N}\right]$.

Now we can prove that the Stanley conjecture holds for ideals of mixed products. It should be noticed that the following case $L=\left(I_{q} J_{t}\right) S=I_{q} S \cap J_{t} S$ was done in [7, 10], especially [6, Corollary 1.12 and Theorem 2.1]. For the completion, we give a direct proof.

Theorem 4.1. Let $L \subset S$ be a monomial ideal of mixed products. Then

(a) $\operatorname{sdepth}_{S}(L) \geq \operatorname{depth}_{S}(L)$;

(b) $\operatorname{sdepth}_{S}(S / L) \geq \operatorname{depth}_{S}(S / L)$.

Proof. Use the notation as above, and set $S_{1}=K\left[x_{1}, \ldots, x_{n}\right]$ and $S_{2}=K\left[x_{n+1}, \ldots, x_{N}\right]$. We will prove (a) and (b) according to $L$ 's following forms:

(1) $I_{q} S, J_{t} S$;

(2) $\left(I_{q} J_{t}\right) S$

(3) $\left(I_{q}+J_{t}\right) S$;

(4) $\left(I_{q} J_{r}+I_{s}\right) S$;

(5) $\left(I_{q} J_{r}+I_{s} J_{t}\right) S$,

where $1 \leq q<s \leq n, 1 \leq t<r \leq N-n$. 
(1) When $L=I_{q} S$ or $J_{t} S$, it is clear from Lemma 2.4 and Lemma $2.1(1)$.

(2) By Proposition 3.1 and Lemma 2.1 (2), we have

$$
\begin{aligned}
& \operatorname{sdepth}_{S}\left(\left(I_{q} J_{t}\right) S\right) \geq \operatorname{sdepth}_{S_{1}}\left(I_{q}\right)+\operatorname{sdepth}_{S_{2}}\left(J_{t}\right) \\
& \geq q+t=\operatorname{depth}_{S}\left(\left(I_{q} J_{t}\right) S\right) ; \operatorname{sdepth}_{S}\left(S /\left(I_{q} J_{t}\right) S\right) \\
& \geq \operatorname{sdepth}_{S_{2}}\left(S_{2} / J_{t}\right)+\min \left\{\operatorname{sdepth}_{S_{1}}\left(I_{q}\right), \operatorname{sdepth}_{S_{1}}\left(S_{1} / I_{q}\right)+1\right\} \\
& \geq t-1+\min \{q, q-1+1\} \\
& =q+t-1=\operatorname{depth}_{S}\left(S /\left(I_{q} J_{t}\right) S\right) .
\end{aligned}
$$

(3) By Proposition 3.2, Lemma 2.3 (3) and Lemma 2.1 (3), we have

$$
\begin{aligned}
& \operatorname{sdepth}_{S}\left(\left(I_{q}+J_{t}\right) S\right) \\
& \geq \operatorname{sdepth}_{S_{2}}\left(J_{t}\right)+\min \left\{\operatorname{sdepth}_{S_{1}}\left(I_{q}\right), \operatorname{sdepth}_{S_{1}}\left(S_{1} / I_{q}\right)\right\} \\
& \geq t+\{q, q-1\}=q+t-1 \\
& =\operatorname{depth}_{S}\left(\left(I_{q}+J_{t}\right) S\right) ; \operatorname{sdepth}_{S}\left(S /\left(I_{q}+J_{t}\right) S\right) \\
& \geq \operatorname{sdepth}_{S_{1}}\left(S_{1} / I_{q}\right)+\operatorname{sdepth}_{S_{2}}\left(S_{2} / J_{t}\right) \\
& =q-1+t-1=q+t-2 \\
& =\operatorname{depth}_{S}\left(S /\left(I_{q}+J_{t}\right) S\right) .
\end{aligned}
$$

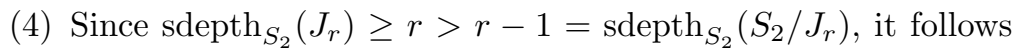
from Proposition 3.3 (1) and Lemma 2.1 (4) that

$$
\begin{aligned}
& \operatorname{sdepth}_{S}\left(\left(I_{q} J_{r}+I_{s}\right) S\right) \\
& \geq \operatorname{sdepth}_{S_{2}}\left(S_{2} / J_{r}\right)+\min \left\{\operatorname{sdepth}_{S_{1}}\left(I_{q}\right)+1, \operatorname{sdepth}_{S_{1}}\left(I_{s}\right)\right\} \\
& \geq r-1+\min \{q+1, s\}=q+r \\
& =\operatorname{depth}_{S}\left(\left(I_{q} J_{r}+I_{s}\right) S\right) .
\end{aligned}
$$

Since $\operatorname{sdepth}_{S_{1}}\left(S_{1} / I_{s}\right)=s-1>q-1=\operatorname{sdepth}_{S_{1}}\left(S_{1} / I_{q}\right)$, it follows from Proposition 3.3 (2) and Lemma 2.1 (4) that

$$
\begin{aligned}
& \operatorname{sdepth}_{S}\left(S /\left(I_{q} J_{r}+I_{s}\right) S\right) \\
& \geq \operatorname{sdepth}_{S_{1}}\left(S_{1} / I_{q}\right) \\
& \quad+\min \left\{\operatorname{sdepth}_{S_{2}}\left(J_{r}\right), \operatorname{sdepth}_{S_{2}}\left(S_{2} / J_{r}\right)+1\right\} \\
& \quad \geq q-1+\min \{r, r-1+1\}=q+r-1 \\
& =\operatorname{depth}_{S}\left(S /\left(I_{q} J_{r}+I_{s}\right) S\right) .
\end{aligned}
$$


(5) Note that $\operatorname{sdepth}_{S_{1}}\left(I_{q} / I_{s}\right) \geq q$ by [4, Proposition 3.1]. Then, from Proposition 3.4 (1) and Lemma 2.1 (5), we obtain

$$
\begin{aligned}
& \operatorname{sdepth}_{S}\left(\left(I_{q} J_{r}+I_{s} J_{t}\right) S\right) \\
& \geq \min \left\{\operatorname{sdepth}_{S_{1}}\left(I_{s}\right)+\operatorname{sdepth}_{S_{2}}\left(J_{t}\right),\right. \\
& \left.\quad \operatorname{sdepth}_{S_{1}}\left(I_{q} / I_{s}\right)+\operatorname{sdepth}_{S_{2}}\left(J_{r}\right)\right\} \\
& \geq \min \{s+t, q+r\} \\
& =\operatorname{depth}_{S}\left(\left(I_{q} J_{r}+I_{s} J_{t}\right) S\right) .
\end{aligned}
$$

Since $\operatorname{sdepth}_{S_{1}}\left(S_{1} / I_{s}\right)=s-1>q-1=\operatorname{sdepth}_{S_{1}}\left(S_{1} / I_{q}\right)$, it follows from Proposition 3.4 (2) and Lemma 2.1 (5) that

$$
\begin{aligned}
& \operatorname{sdepth}_{S}(\left.S /\left(I_{q} J_{r}+I_{s} J_{t}\right) S\right) \\
& \geq \min \left\{\operatorname{sdepth}_{S_{1}}\left(S_{1} / I_{q}\right)\right. \\
& \quad+\min \left\{\operatorname{sdepth}_{S_{2}}\left(J_{r}\right), \operatorname{sdepth}_{S_{2}}\left(S_{2} / J_{r}\right)+1\right\} \\
&\left.\quad \operatorname{sdepth}_{S_{1}}\left(I_{s}\right)+\operatorname{sdepth}_{S_{2}}\left(S_{2} / J_{t}\right)\right\} \\
& \geq \min \{q-1+\min \{r, r-1+1\}, s+t-1\} \\
&=\min \{q+r, s+t\}-1 \\
&=\operatorname{depth}_{S}\left(S /\left(I_{q} J_{r}+I_{s} J_{t}\right) S\right) .
\end{aligned}
$$

It is known that the above theorem holds for any monomial ideal $L$ with $N \leq 5$ or if $L$ is an intersection of four monomial prime ideals (cf., $[\mathbf{8}, \mathbf{9}, \mathbf{1 0}, \mathbf{1 4}])$. The following example demonstrates that the set of ideals of mixed products involves more ideals on which the Stanley conjecture holds.

Example 4.2. Let $S=K\left[x_{1}, \ldots, x_{6}\right]$ be a polynomial ring over a field $K$ and

$$
\begin{aligned}
L= & \left(I_{2} J_{1}+I_{1} J_{2}\right) S=\left(x_{1} x_{2} x_{4}, x_{1} x_{3} x_{4}, x_{2} x_{3} x_{4}, x_{1} x_{2} x_{5}, x_{1} x_{3} x_{5},\right. \\
& x_{2} x_{3} x_{5}, x_{1} x_{2} x_{6}, x_{1} x_{3} x_{6}, x_{2} x_{3} x_{6}, x_{1} x_{4} x_{5}, x_{2} x_{4} x_{5}, x_{3} x_{4} x_{5}, \\
& \left.x_{1} x_{4} x_{6}, x_{2} x_{4} x_{6}, x_{3} x_{4} x_{6}, x_{1} x_{5} x_{6}, x_{2} x_{5} x_{6}, x_{3} x_{5} x_{6}\right)
\end{aligned}
$$

an ideal of mixed products, where $I_{2}$ (respectively, $I_{1}$ ) is the squarefree monomial ideal of $K\left[x_{1}, x_{2}, x_{3}\right]$ generated by all the square-free monomials of degree 2 (respectively, 1 ) and $J_{2}$ (respectively, $J_{1}$ ) is the square-free monomial ideal of $K\left[x_{4}, x_{5}, x_{6}\right]$ generated by all the square- 
free monomials of degree 2 (respectively, 1 ). Note that

$$
\begin{aligned}
L= & \left(x_{1}, x_{2}, x_{3}\right) \cap\left(x_{4}, x_{5}, x_{6}\right) \cap\left(x_{1}, x_{3}, x_{5}, x_{6}\right) \cap\left(x_{2}, x_{3}, x_{5}, x_{6}\right) \\
& \cap\left(x_{1}, x_{3}, x_{4}, x_{5}\right) \cap\left(x_{2}, x_{3}, x_{4}, x_{5}\right) \cap\left(x_{1}, x_{2}, x_{4}, x_{5}\right) \\
& \cap\left(x_{1}, x_{2}, x_{4}, x_{6}\right) \cap\left(x_{1}, x_{2}, x_{5}, x_{6}\right) \\
& \cap\left(x_{1}, x_{3}, x_{4}, x_{6}\right) \cap\left(x_{2}, x_{3}, x_{4}, x_{6}\right)
\end{aligned}
$$

is an intersection of prime ideals of $S$ such that each prime ideal is contained in the sum of the remaining prime ideals, not satisfying $[\mathbf{1 0}$, Theorem 2.4].

Acknowledgments. The second author is grateful to the Department of Mathematics and Computer Science of the University of Messina for its hospitality. The authors are also grateful to the referee for his/her good comments.

\section{REFERENCES}

1. W. Bruns and J. Herzog, Cohen-Macaulay rings, Cambridge University Press, Cambridge, 1997.

2. M. Cimpoeas, Several inequalities regarding Stanley depth, Roum. J. Math. Comp. Sci. 2 (2012), 28-40.

3. J. Herzog, D. Popescu and M. Vladoiu, Stanley depth and size of a monomial ideal, Proc. Amer. Math. Soc. 140 (2012), 493-504.

4. J. Herzog, M. Vladoiu and X. Zheng, How to compute the Stanley depth of a monomial ideal, J. Alg. 322 (2009), 3151-3169.

5. C. Ionescu and G. Rinaldo, Some algebraic invariants related to mixed product ideals, Arch. Math. 91 (2008), 20-30.

6. M. Ishaq, Values and bounds of the Stanley depth, Carpathian J. Math. 27 (2011), 217-224.

7. A. Popescu, Special Stanley decompositions, Bull. Math. Soc. Sci. Math. Roum. 53 (2010), 363-372.

8. D. Popescu, An inequality between depth and Stanley depth, Bull. Math. Soc. Sci. Math. Roum. 52 (2009), 377-382.

9. _ Stanley depth of multigraded modules, J. Alg. 321 (2009), 2782-2797.

10. Stanley conjecture on intersections of four monomial prime ideals, Comm. Alg. 41 (2013), 4351-4362.

11. A. Rauf, Depth and Stanley depth of multigraded module, Comm. Alg. 38 (2010), 773-784.

12. G. Restuccia and R. Villarreal, On the normality of monomial ideals of mixed products, Comm. Alg. 29 (2001), 3571-3580. 
13. R.P. Stanley, Linear Diophantine equations and local cohomology, Invent. Math. 68 (1982), 175-193.

14. Z. Tang, Stanley depths of certain Stanley-Reisner rings, J. Alg. 409 (2014), 430-443.

15. R.H. Villarreal, Monomial algebras, Dekker, New York, 2001.

Dipartimento di Matematica e Informatica, Universitá di Messina, Viale Ferdinando Stagno d'Alcontres 31, 98166 Messina, Italy

Email address: gaetana.restuccia@unime.it

Department of Mathematics, Suzhou University, Suzhou 215006, P.R. China Email address: zmtang@suda.edu.cn

Dipartimento di Matematica e Informatica, Universitá di Messina, Viale Ferdinando Stagno d'Alcontres 31, 98166 Messina, Italy

Email address: rosanna.utano@unime.it 